\title{
Influence of Non-linear Boussinesq Approximation on Natural Convective Flow of a Power-Law Fluid along an Inclined Plate under Convective Thermal Boundary Condition
}

https://doi.org/10.1515/nleng-2017-0138

Received July 26, 2017; revised March 23, 2018; accepted May 5, 2018.

\begin{abstract}
In the present investigation, a problem of natural convective flow of a non-Newtonian power-law fluid over an inclined plate saturated in a non-Darcy porous medium is considered. Also, the nonlinear Boussinesq approximation and convective thermal boundary condition are taken into account to address heat and mass transfer phenomena of thermal systems which are operated at moderate and very high temperatures. The steady-state boundary layer equations are non-dimensionalized into non-similar form and then solved numerically by the local non-similarity method with successive linearisation method (SLM). The effects of various physical parameters on the fluid flow, heat and mass transfer characteristics are depicted graphically and analysed in detail.
\end{abstract}

Keywords: Non-linear Boussinesq Approximation; NonDarcy Porous Medium; Convective Boundary Condition; Successive Linearization Method; Power-Law Fluid

\section{Introduction}

Many problems related to energy and geophysical industries (such as thermal insulation, geophysical flows, petroleum resource, polymer processing, etc.) requires the analysis of the free convective flow of non-Newtonian fluids in a porous medium. Since, the majority of the real fluids (cosmetic products, grease, body fluids, and much more) exhibits a non-Newtonian behaviour and

\footnotetext{
*Corresponding Author: Ch. RamReddy, Department of Mathematics, National Institute of Technology Warangal-506004, India, E-mail: chittetiram@gmail.com

P. Naveen, D. Srinivasacharya, Department of Mathematics, National Institute of Technology Warangal-506004, India
}

the study of non-Newtonian fluids in a porous matrix are very different from that of Newtonian fluids in porous media. Various fluid models have been suggested and studied to describe the dynamics of non-Newtonian fluids. Among them, Ostwald-de Waele power-law fluid is one, and it has strong applications in many engineering industries. Because it characterizes the flow pattern of certain non-Newtonian fluids which contradict Newton's law of viscosity like polymer melt and glass, etc. Several authors, Shenoy [1, 2], Gorla and Kumari [3], Kumari and Nath [4], Cheng [5], Kairi and RamReddy [6] to mention but a few, have studied the free/mixed convective transport over different surface geometries in a power-law fluid-saturated porous media.

Heat transfer analysis with convective thermal boundary condition is an important and useful consideration in the gas turbines, nuclear plants, heat exchangers related industries, due to the realistic nature of this condition. In this mechanism, heat is supplied to the convecting fluid through a bounding surface with a finite heat capacity, which provides a convective heat transfer coefficient. In view of these applications, Makinde [7] carried out an analysis to discuss the steady laminar MHD flow over a moving vertical plate with convective boundary condition. Khan and Gorla [8] considered the Robin boundary condition(convective boundary condition) in the boundary layer analysis of mixed convection of water at $4^{\circ} \mathrm{C}$ along a vertical surface. In the recent times, the thermal radiation and Joule heating on MHD micropolar fluid has been discussed by Ramzan et al. [9] under the consideration of convective surface boundary conditions.

Some of the thermal systems such as those encountered in reactor safety, combustion and solar collectors operate at moderate to very high temperatures and in such situations, the density relation with temperature and concentration may become nonlinear. This nonlinear variation in temperature-concentration-dependent 
density relation (to be specific, nonlinear Boussinesq approximation or nonlinear convection) gives a strong influence to the fluid flow characteristics (for more details see the work of Barrow and Sitharamarao [10], Vajravelu and Sastri [11]). Partha [12] studied numerically the convective heat and mass transfer in the influence of nonlinear Boussinesq approximation, dispersion, and cross-diffusion effects on the non-Darcy fluid flow over a vertical surface. A Darcy-Forchheimer model considered in the analysis of nonlinear convection and thermophoresis in a regular fluid by Kameswaran et al. [13] and concluded that the boundary layer thickness of temperature and concentration is reduced with enhancing values of nonlinear convection parameters.

Nowadays, most of the researchers are interested to analyze the free convection from an inclined surface in both theoretical and experimental works. Because, free convection heat and mass transfer through an inclined surface are frequently encountered in natural and engineering devices such as solar water heaters, electrical systems, iron removal, brine clarification, etc. It has been shown that the inclination of the flat plate to the vertical reduces the drag force which favors the flow through the medium. Chamka et al. [14] addressed the importance of angle inclination in the analysis of natural convective flow along an inclined surface embedded in a porous medium. Influence of cross-diffusion and variable thermal conductivity are considered on MHD convective power-law fluid flow past an inclined plate by $\mathrm{Pal}$ and Chatterjee [15]. Further, Murthy et al. [16] analyzed the influence of Soret and inclination of angle on the double-diffusive natural convective flow of nanofluid with Forchheimer model.

Based on the previous studies, it is relevant to discuss the free convective flow of a power-law fluid along an inclined plate with a Biot number effect. Also, the nonlinear Boussinesq approximation is considered in the formulation of fluid flow equations with a Darcy-Forchhiemer's model. This kind of investigation is useful in the mechanism of combustion, solar collectors which are performed at high-level temperatures.

\section{Mathematical Modeling}

A two-dimensional, steady, incompressible, free convective flow of power-law fluid along an inclined plate embedded in a non-Darcy porous medium is considered. The semi-infinite plate is inclined about vertical direction with an acute angle $\Omega$, as shown in Fig.1. In this figure, M.B.L is used to represent momentum boundary layer, while T.B.L and C.B.L are used to represent thermal and concentration boundary layers, respectively. The plate is either heated or cooled from left by convection from a fluid of temperature $T_{f}$ with $T_{f}>T_{\infty}$ corresponding to a heated surface and $T_{f}<T_{\infty}$ corresponding to a cooled surface, respectively. It is further assumed that the concentration of the ambient fluid is of uniform magnitude, $C_{\infty}$, the unknown wall concentration of the plate is $C_{w}$. It is assumed that the fluid and the porous medium have constant physical properties except for the density variation in the buoyancy term (which is linear/nonlinear variation with temperature and concentration) in the momentum equation. The fluid flow is moderate, and the permeability of the medium is low so that the Forchheimer flow model is employed to simulate the resistance of the porous medium fibers on the buoyant flow.

A power-law fluid is a type of generalized Newtonian fluid for which the shear stress $\tau_{x y}$ can be expressed as $\tau_{x y}=\mu^{*}\left|\frac{\partial u}{\partial y}\right|^{n-1} \frac{\partial u}{\partial y}$. Here, $\mu^{*}$ is called the consistency coefficient and $n$ is the power-law index. The dimension of $\mu^{*}$ depends on the value of $n$ which is non-dimensional. When $n=1$, the fluid become the Newtonian fluid with a dynamic coefficient of viscosity $\mu^{\star}$. Therefore, deviation of $n$ from a unity indicates the degree of deviation from Newtonian fluid behavior. That is for $n<1$, the fluid is pseudoplastic and for $n>1$, the fluid is dilatant. The governing equations for the flow, heat and mass transfer of a powerlaw fluid saturated non-Darcy porous medium (Shenoy [2], Murthy and Singh [17] and Chen [18]) are given by

$$
\begin{gathered}
\frac{\partial u}{\partial x}+\frac{\partial v}{\partial y}=0 \\
u^{n}+\frac{b \sqrt{K_{p}}}{v} u^{2}=-\frac{K_{p}}{\mu}\left(\frac{\partial p}{\partial x}+\rho g^{\star} \cos \Omega\right) \\
v^{n}+\frac{b \sqrt{K_{p}}}{v} v^{2}=-\frac{K_{p}}{\mu}\left(\frac{\partial p}{\partial y}\right) \\
u \frac{\partial T}{\partial x}+v \frac{\partial T}{\partial y}=\alpha\left(\frac{\partial^{2} T}{\partial x^{2}}+\frac{\partial^{2} T}{\partial y^{2}}\right) \\
u \frac{\partial C}{\partial x}+v \frac{\partial C}{\partial y}=D\left(\frac{\partial^{2} C}{\partial x^{2}}+\frac{\partial^{2} C}{\partial y^{2}}\right)
\end{gathered}
$$

As the fundametal study, several researchers anlysed the solution of Eqs. (1)-(5) by considering the following linear Boussinesq approximation, in which density is expressed as a linear function of the temperature and concentration.

$$
\rho=\rho_{\infty}\left[1-\beta_{0}\left(T-T_{\infty}\right)-\beta_{2}\left(C-C_{\infty}\right)\right]
$$


But, the presence of temperature variation, radiation, inertia, or presence of different densities and heat released by viscous dissipation induce significant changes in density gradients, and hence the density, temperature and concentration relationship become nonlinear. Due to this, the results of fluid flow problem with linear Boussinesq approximation are inaccurate. To explain this situation, Partha [12] investigated the natural nonlinear convection in a non-Darcy porous medium using the following non-linear relationship among temperatureconcentration-dependent density (also known as nonlinear Boussinesq approximation)

$$
\begin{aligned}
\rho= & \rho_{\infty}\left[1-\beta_{0}\left(T-T_{\infty}\right)-\beta_{1}\left(T-T_{\infty}\right)^{2}-\beta_{2}\left(C-C_{\infty}\right)\right. \\
& \left.-\beta_{3}\left(C-C_{\infty}\right)^{2}\right]
\end{aligned}
$$

along with the associated boundary conditions

$$
\begin{array}{r}
v=0,-k_{f} \frac{\partial T}{\partial y}=h_{f}\left(T_{f}-T\right), \quad C=C_{w} \quad \text { at } \quad y=0 \\
u=0, \quad T=T_{\infty}, \quad C=C_{\infty} \quad \text { as } \quad y \rightarrow \infty
\end{array}
$$

where $(u, v)$ are the Darcian velocities, $\rho$ is the density, $p$ is the pressure, $K_{p}$ is the permeability, $k_{f}$ is the thermal conductivity, $C$ is the concentration, $D$ is the solutal diffusivity, $v$ is the kinematic viscosity, $h_{f}$ is the convective heat transfer coefficient, $\alpha$ is the thermal diffusivity, $b$ is the empirical constant, $\Omega$ is the inclination of angle, $g^{\star}$ is the acceleration due to gravity, and $T$ is the temperature, respectively. Further, the first and second order thermal and solutal coefficients are taken as $\beta_{0}$ and $\beta_{1}, \beta_{2}$ and $\beta_{3}$, respectively. Furthur, the suffix $w$ and $\infty$ indicate the conditions at the wall and at the outer edge of the boundary layer, respectively.

Experimental and numerical studies on convective heat transfer in porous media show that thermal boundary layers exist adjacent to the heated or cooled bodies. When the thermal boundary layer is thin (i.e., $x \gg y \sim \delta_{T}$, $\delta_{T}$ is the boundary layer thickness), boundary layer approximations analogous to classical boundary layer theory can be applied (Nield and Bejan [19]). Near the boundary, the normal component of seepage velocity is small compared with the other component of the seepage velocity and the derivatives of any quantity in the normal direction are large compared with derivatives of the quantity in the direction of the wall. Now, making use of the boundary layer assumptions, nonlinear Boussinesq approximations and eliminating pressure gradient from the momentum equations, the governing equations (2)-(5) reduces to

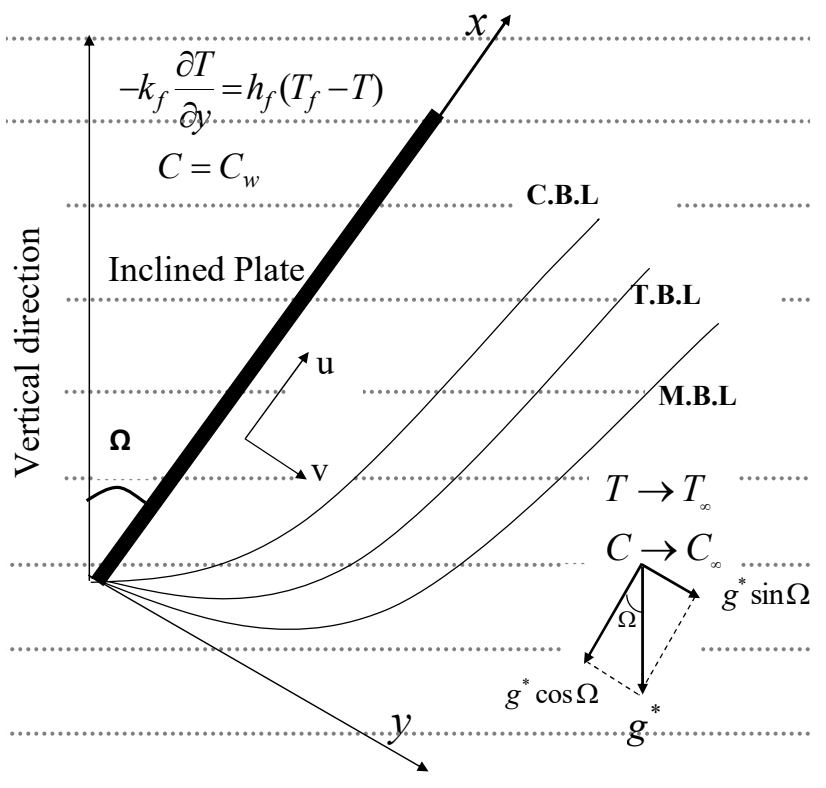

Fig. 1: Schematic diagram of the problem

$$
\begin{aligned}
& \frac{\partial u^{n}}{\partial y}+\frac{b \sqrt{K_{p}}}{v} \frac{\partial u^{2}}{\partial y}=\frac{K_{p} g^{\star}}{v} \\
& \left\{\left[\beta_{0}+2 \beta_{1}\left(T-T_{\infty}\right)\right] \frac{\partial T}{\partial y}+\left[\beta_{2}+2 \beta_{3}\left(C-C_{\infty}\right)\right] \frac{\partial C}{\partial y}\right\} \cos \Omega
\end{aligned}
$$

$$
\begin{aligned}
& u \frac{\partial T}{\partial x}+v \frac{\partial T}{\partial y}=\alpha \frac{\partial^{2} T}{\partial y^{2}} \\
& u \frac{\partial C}{\partial x}+v \frac{\partial C}{\partial y}=D \frac{\partial^{2} C}{\partial y^{2}}
\end{aligned}
$$

By defining stream function $\psi(x, y)$ as $u=\frac{\partial \psi}{\partial y}$ and $v=-\frac{\partial \psi}{\partial x}$, the continuity equation (1) is automatically satisfied. To convert the system of dimensional equations (9) - (11) into system of the equations in the non-dimensional form, we considered the following dimensionless nonsimilarity transformations

$$
\begin{aligned}
& \xi=\frac{x}{L}, \quad \eta=\frac{y}{L} R a^{\frac{1}{2}} \xi^{\frac{-1}{2}}, \quad \psi(\xi, \eta)=\alpha \xi^{\frac{1}{2}} R a^{\frac{1}{2}} f(\xi, \eta) \\
& T(\xi, \eta)=T_{\infty}+\left(T_{f}-T_{\infty}\right) \theta(\xi, \eta), \\
& C(\xi, \eta)=C_{\infty}+\left(C_{w}-C_{\infty}\right) \phi(\xi, \eta)
\end{aligned}
$$

where $R a=\frac{L}{\alpha}\left[\frac{\rho_{\infty} K_{p} g^{*} \beta_{0}\left(T_{f}-T_{\infty}\right)}{\mu^{*}}\right]^{1 / n}$ is the global Rayleigh number,

Substituting the transformations (12) into Eqs. (9) (11), we obtain the following momentum, energy and con- 
centration equations

$$
\begin{gathered}
n\left(f^{\prime}\right)^{n-1} f^{\prime \prime}+2 G r^{*} f^{\prime} f^{\prime \prime} \\
=\left[\left(1+2 \alpha_{1} \theta\right) \theta^{\prime}+\mathcal{B}\left(1+2 \alpha_{2} \phi\right) \phi^{\prime}\right] \cos \Omega \\
\theta^{\prime \prime}+\frac{1}{2} f \theta^{\prime}=\xi\left(f^{\prime} \frac{\partial \theta}{\partial \xi}-\frac{\partial f}{\partial \xi} \theta^{\prime}\right) \\
\frac{1}{L e} \phi^{\prime \prime}+\frac{1}{2} f \phi^{\prime}=\xi\left(f^{\prime} \frac{\partial \phi}{\partial \xi}-\frac{\partial f}{\partial \xi} \phi^{\prime}\right)
\end{gathered}
$$

Dimensionless form of boundary conditions (8) become

$$
\begin{aligned}
& f(\xi, 0)=-2 \xi\left(\frac{\partial f}{\partial \xi}\right)_{\eta=0}, \\
& \theta^{\prime}(\xi, 0)=-B i \xi^{\frac{1}{2}}[1-\theta(\xi, 0)], \phi(\xi, 0)=1, \\
& f^{\prime}(\xi, \infty)=0, \theta(\xi, \infty)=0, \phi(\xi, \infty)=0 .
\end{aligned}
$$

In usual definitions,

$$
G r^{\star}=b\left[\rho_{\infty}{ }^{2} K_{p}^{2}\left[g^{\star} \beta_{0}\left(T_{f}-T_{\infty}\right)\right]^{2-n} / \mu^{\star 2}\right]^{1 / n}
$$

is the modified Grashof number, $\xi$ is the streamwise coordinate, $S c=v / D$ is the Schmidt number, $\mathcal{B}=\beta_{2}\left(C_{w}-\right.$ $\left.C_{\infty}\right) /\left(\beta_{0}\left(T_{f}-T_{\infty}\right)\right)$ is the Buoyancy ratio, $\alpha_{1}=\beta_{1}\left(T_{f}-\right.$ $\left.T_{\infty}\right) / \beta_{0}$ is the nonlinear density-temperature parameter, $L e=(\alpha / D)$ is the diffusivity ratio, $\mathrm{Pr}=v / \alpha$ is the Prandtl number, $B i=h_{f} L /\left(k_{f} R a^{1 / 2}\right)$ is the Biot number, and $\alpha_{2}=$ $\beta_{3}\left(C_{w}-C_{\infty}\right) / \beta_{2}$ is the nonlinear density-concentration parameter, respectively.

The non-dimensional shear stress $C_{f}=\frac{2 \tau_{w}}{\rho u_{\star}^{2}}$, Nusselt number $N u_{x}=\frac{-x}{\left(T_{f}-T_{\infty}\right)}\left[\frac{\partial T}{\partial y}\right]_{y=0}$ and Sherwood number $S h_{x}=\frac{-x}{\left(C_{w}-C_{\infty}\right)}\left[\frac{\partial C}{\partial y}\right]_{y=0}$ are given by

$$
\begin{aligned}
& \frac{\operatorname{Pr} P e^{2}}{2 R a^{\frac{3}{2}}} C_{f}=\xi^{\frac{-1}{2}} f^{\prime \prime}(\xi, 0), N u R a^{\frac{-1}{2}}=-\xi^{\frac{1}{2}} \theta^{\prime}(\xi, 0), \\
& \operatorname{Sh} R a^{\frac{-1}{2}}=-\xi^{\frac{1}{2}} \phi^{\prime}(\xi, 0) .
\end{aligned}
$$

\section{Numerical solutions}

The set of nonlinear partial differential equations (13)-(15) with boundary conditions (16) have been solved numerically by using the Successive Linearisation Method(SLM) (see. Makukula et al. [20], Awad et al. [21], Khidir et al. [22]) together with Local non-similarity procedure (Sparrow and $\mathrm{Yu}$ [23], Minkowycz and Cheng [24]).

\subsection{Local non-similarity procedure}

The initial approximate solution can be found from local similarity equations for a particular case of $\xi \ll 1$, for this the terms containing $\xi \frac{\partial}{\partial \xi}$ are supposed to be negligible. Then the local similarity equations from (13)-(16) are

$$
\begin{aligned}
& n\left(f^{\prime}\right)^{n-1} f^{\prime \prime}+2 G r^{\star} f^{\prime} f^{\prime \prime} \\
& =\left[\left(1+2 \alpha_{1} \theta\right) \theta^{\prime}+\mathcal{B}\left(1+2 \alpha_{2} \phi\right) \phi^{\prime}\right] \cos \Omega
\end{aligned}
$$

$$
\begin{gathered}
\theta^{\prime \prime}+\frac{1}{2} f \theta^{\prime}=0 \\
\frac{1}{L e} \phi^{\prime \prime}+\frac{1}{2} f \phi^{\prime}=0
\end{gathered}
$$

The transformed boundary conditions are

$$
\begin{array}{r}
f(\xi, 0)=0, \theta^{\prime}(\xi, 0)=-B i \xi^{\frac{1}{2}}[1-\theta(\xi, 0)], \phi(\xi, 0)=1, \\
f^{\prime}(\xi, \infty)=0, \theta(\xi, \infty)=0, \phi(\xi, \infty)=0 .
\end{array}
$$

In order to overcome the limitations imposed by local-similarity method, the local non-similar boundary-layer equations will now be derived. The two-equation model will be first derived. Let $\frac{\partial f(\xi, \eta)}{\partial \xi}=U(\xi, \eta), \frac{\partial \theta(\xi, \eta)}{\partial \xi}=V(\xi, \eta), \frac{\partial \phi(\xi, \eta)}{\partial \xi}$

$W(\xi, \eta)$. Introducing these functions into Eqs. (13)(16), then we get first set of auxiliary equations and boundary conditions:

$$
\begin{gathered}
n\left(f^{\prime}\right)^{n-1} f^{\prime \prime}+2 G r^{\star} f^{\prime} f^{\prime \prime} \\
=\left[\left(1+2 \alpha_{1} \theta\right) \theta^{\prime}+\mathcal{B}\left(1+2 \alpha_{2} \phi\right) \phi^{\prime}\right] \cos \Omega \\
\theta^{\prime \prime}+\frac{1}{2} f \theta^{\prime}=\xi\left(V f^{\prime}-U \theta^{\prime}\right) \\
\frac{1}{L e} \phi^{\prime \prime}+\frac{1}{2} f \phi^{\prime}=\xi\left(W f^{\prime}-U \phi^{\prime}\right)
\end{gathered}
$$

The transformed boundary conditions are

$$
\begin{aligned}
& f(\xi, 0)=-2 \xi U(\xi, \eta), \theta^{\prime}(\xi, 0)=-B i \xi^{\frac{1}{2}}[1-\theta(\xi, 0)], \\
& \phi(\xi, 0)=1, \\
& f^{\prime}(\xi, \infty)=0, \theta(\xi, \infty)=0, \phi(\xi, \infty)=0 .
\end{aligned}
$$

Differentiating the above Eqs. (22)-(25) with respect to $\xi$ and neglecting the terms involving the derivative functions $U, V, W$ with respect to $\xi$, we obtain second set of auxiliary equations and boundary conditions:

$n(n-1)\left(f^{\prime}\right)^{n-2} U^{\prime} f^{\prime \prime}+n\left(f^{\prime}\right)^{n-1} U^{\prime \prime}+2 G r^{\star}\left(U^{\prime} f^{\prime \prime}+f^{\prime} U^{\prime \prime}\right)=$
$\left[V^{\prime}+2 \alpha_{1}\left(V \theta^{\prime}+\theta V^{\prime}\right)+\mathcal{B}\left(W^{\prime}+2 \alpha_{2}\left(W \phi^{\prime}+\phi W^{\prime}\right)\right)\right] \cos \Omega$ 


$$
\begin{aligned}
V^{\prime \prime}+\frac{3}{2} U \theta^{\prime}+\frac{1}{2} f V^{\prime}-f^{\prime} V & =\xi\left(V U^{\prime}-V^{\prime} U\right) \\
\frac{1}{L e} W^{\prime \prime}+\frac{3}{2} U \phi^{\prime}+\frac{1}{2} f W^{\prime}-f^{\prime} W & =\xi\left(W U^{\prime}-W^{\prime} U\right)
\end{aligned}
$$

The corresponding boundary conditions are

$$
\begin{aligned}
& U(\xi, 0)=0, V^{\prime}(\xi, 0)=B i \xi^{\frac{1}{2}} V(\xi, 0)+\frac{1}{2} B i \xi^{\frac{-1}{2}}[\theta(\xi, 0)-1], \\
& W(\xi, 0)=0, \\
& U^{\prime}(\xi, \infty)=0, V(\xi, \infty)=0, W(\xi, \infty)=0
\end{aligned}
$$

The two-equation model involves six coupled boundary layer equations that need to be solved simultaneously in conjunction with a set of boundary conditions. Although the solution to the two-equation model provides information on six parameters, it is only $f, \theta$ and $\phi$, and their derivatives that are of primary interest since they are the ones meant to the physical problem. The local nonsimilarity transformation preserves the non-similar terms in original governing equations and boundary conditions with only part of the non-similar terms dropped from its auxiliary equations. Since the original governing equations remain intact, the local non-similarity method is expected to be more accurate than the local-similarity solution

\subsection{Successive Linearization Method}

The coupled nonlinear differential equations (22) - (24) and (26) - (28) along with the boundary conditions (25) and (29) are solved using Successive Linearization Method. First, it linearise the nonlinear equations and then it utilizes Chebyshev collocation method for the approximate solution.

\subsubsection{Successive Linearization}

Let us consider an independent vector $\mathbb{Q}(\eta)=$ $[f(\eta), \theta(\eta), \phi(\eta), U(\eta), V(\eta), W(\eta)]$ and assume that it can be represented as

$$
\mathbb{Q}(\eta)=\mathbb{Q}_{k}(\eta)+\sum_{r=0}^{k-1} \mathbb{Q}_{r}(\eta)
$$

where $\mathbb{Q}_{k}(\eta), k=1,2,3 \ldots .$. , are unknown functions and $\mathbb{Q}_{m}(\eta),(0 \leq m \leq k-1)$ are successive approximations which are obtained by recursively solving the linear part of the equation system that results from substituting firstly
Eq.(30) in Eqs.(22)- (29). The main assumption of the SLM is that $\mathbb{Q}_{k}$ become increasingly smaller when $k$ becomes large, that is

$$
\lim _{k \rightarrow \infty} \mathbb{Q}_{k}=0
$$

The initial guesses $\mathbb{Q}_{0}(\eta)$ which are chosen to satisfy the boundary conditions (25) and (29). Thus, starting from the initial guesses, the subsequent solutions $\mathbb{Q}_{k}(\eta)$ are obtained by successively solving the linearized form of the equations which are obtained by substituting equation (30) in Eqs.(22)- (29) and neglecting the nonlinear terms containing $\mathbb{Q}_{k}(\eta)(k \geq 1)$ and its derivatives. The linearized equations to be solved are

$$
\begin{aligned}
& \tilde{p}_{1, k-1} f_{k}{ }^{\prime \prime}+\tilde{p}_{2, k-1} f_{k}{ }^{\prime}+\tilde{p}_{3, k-1} \theta_{k}{ }^{\prime}+\tilde{p}_{4, k-1} \theta_{k}+\tilde{p}_{5, k-1} \phi_{k}{ }^{\prime} \\
& +\tilde{p}_{6, k-1} \phi_{k}=\tilde{z}_{1, k-1} \\
& \quad \tilde{q}_{1, k-1} f_{k}+\tilde{q}_{2, k-1} \theta_{k}^{\prime \prime}+\tilde{q}_{3, k-1} \theta_{k}^{\prime}+\tilde{q}_{4, k-1} U_{k} \\
& \quad+\tilde{q}_{5, k-1} V_{k}=\tilde{z}_{2, k-1} \\
& \tilde{a}_{1, k-1} f_{k}+\tilde{a}_{2, k-1} \phi_{k}^{\prime \prime}+\tilde{a}_{3, k-1} \phi_{k}^{\prime}+\tilde{a}_{4, k-1} U_{k} \\
& +\tilde{a}_{5, k-1} W_{k}=\tilde{z}_{3, k-1}
\end{aligned}
$$

$$
\begin{aligned}
& \tilde{b}_{1, k-1} f_{k}{ }^{\prime \prime}+\tilde{b}_{2, k-1} f_{k}{ }^{\prime}+\tilde{b}_{3, k-1} \theta_{k}{ }^{\prime}+\tilde{b}_{4, k-1} \theta_{k}+\tilde{b}_{5, k-1} \phi_{k}{ }^{\prime} \\
& +\tilde{b}_{6, k-1} \phi_{k}+\tilde{b}_{7, k-1} U^{\prime \prime}{ }_{k}+\tilde{b}_{8, k-1} U^{\prime}{ }_{k}+\tilde{b}_{9, k-1} V_{k}^{\prime} \\
& +\tilde{b}_{10, k-1} V_{k}+\tilde{b}_{11, k-1} W^{\prime}{ }_{k}+\tilde{b}_{12, k-1} W_{k}=\tilde{z}_{4, k-1} \\
& \tilde{c}_{1, k-1} f_{k}+\tilde{c}_{2, k-1} \theta_{k}{ }^{\prime}+\tilde{c}_{3, k-1} U_{k}^{\prime}+\tilde{c}_{4, k-1} U_{k}+\tilde{c}_{5, k-1} V_{k}{ }^{\prime \prime} \\
& +\tilde{c}_{6, k-1} H_{k}{ }^{\prime}+\tilde{c}_{7, k-1} V_{k}=\tilde{z}_{5, k-1} \\
& \tilde{d}_{1, k-1} f_{k}+\tilde{d}_{2, k-1} \phi_{k}{ }^{\prime}+\tilde{d}_{3, k-1} U_{k}^{\prime}+\tilde{d}_{4, k-1} U_{k}+\tilde{d}_{5, k-1} W_{k}{ }^{\prime \prime} \\
& +\tilde{d}_{6, k-1} W_{k}{ }^{\prime}+\tilde{d}_{7, k-1} W_{k}=\tilde{z}_{6, k-1}
\end{aligned}
$$

The linearised boundary conditions are

$$
\begin{aligned}
& f_{k}(0)=f_{k}{ }^{\prime}(0)=f_{k}{ }^{\prime}(\infty)=0, B i \xi^{\frac{1}{2}} \theta_{k}(0)+\theta_{k}{ }^{\prime}(0)=0, \\
& \theta_{k}(\infty)=0, \phi_{k}(0)=\phi_{k}(\infty)=0, \\
& U_{k}(0)=U_{k}{ }^{\prime}(0)=U_{k}{ }^{\prime}(\infty)=0, \\
& -\frac{1}{2} B i \xi^{\frac{-1}{2}} \theta_{k}(0)+V^{\prime}{ }_{k}(0)-B i \xi^{\frac{1}{2}} V_{k}(0)=0, \\
& V_{k}(\infty)=0, W_{k}(0)=W_{k}(\infty)=0
\end{aligned}
$$

Here the coefficient parameters $\tilde{p}_{s, k-1}, \tilde{q}_{s, k-1}$, $\tilde{a}_{s, k-1}, \tilde{b}_{s, k-1}, \tilde{c}_{s, k-1}, \tilde{d}_{s, k-1}$, and $\tilde{z}_{s, k-1}$ which depend on the $\mathbb{Q}_{0}(\eta)$ and on the $\mathbb{Q}_{k}(\eta)$ derivatives. 


\subsubsection{Chebyshev collocation scheme:}

We solve linearised equations (32) - (37) by an established procedure, namely Chebyshev collocation scheme (Canuto et al. [25]). In the context of numerical implication, the original region $[0, \infty)$ is truncated to $[0, L]$ for large value of $L$, and further the truncated region $[0, L]$ is transformed into $[-1,1]$ using the following mapping

$$
\frac{\eta}{L}=\frac{\tau+1}{2}, \quad-1 \leq \tau \leq 1
$$

In this procedure, The Chebyshev polynomials $T_{r}(\tau)=$ $\cos \left[r \cos ^{-1} \tau\right]$ are used to approximate the unknown functions $\mathbb{Q}_{k}(\eta)$ and these polynomials are collocated at $N+1$ Gauss-Lobatto points in the interval $[-1,1]$ and those are defined as

$$
\tau_{m}=\cos \frac{\pi m}{N}, \quad m=0,1, \ldots, N
$$

The unknown function $\mathbb{Q}_{k}(\eta)$ is imprecise at the collocation points by

$$
\mathbb{Q}_{k}(\tau)=\sum_{r=0}^{N} \mathbb{Q}_{k}\left(\tau_{r}\right) T_{r}\left(\tau_{m}\right), \quad m=0,1, \ldots N
$$

and

$$
\frac{d^{\mathbb{S}}}{d \eta^{\mathbb{S}}} \mathbb{Q}_{k}(\tau)=\sum_{r=0}^{N} \mathbf{D}_{r m}^{\mathbb{S}} \mathbb{Q}_{k}\left(\tau_{r}\right), \quad m=0,1,2, \ldots N
$$

where $\mathcal{D}$ is the Chebyshev spectral derivative matrix such that $\mathbf{D}=(2 / L) \mathcal{D}$ and $\mathbb{S}$ is the order of differentiation. After employing Eqs.(39) - (42) into linearized form of equations (32)- (37), the resultant solution is

$$
\tilde{\mathbf{Y}}_{k}=\tilde{\mathbf{B}}_{k-1}^{-1} \tilde{\mathbf{Z}}_{k-1}
$$

In Eq.(43), $\tilde{\mathbf{B}}_{k-1}$ is a $(6 N+6) \times(6 N+6)$ matrix, $\tilde{\mathbf{Y}}_{k}$ and $\tilde{\mathbf{Z}}_{k-1}$ are $(6 N+1) \times 1$ column vectors defined by

$$
\begin{aligned}
& \tilde{\mathbf{B}}_{k-1}=\left[\tilde{\mathbf{B}}_{i j}\right], i, j=1,2, \ldots 6 \text {, } \\
& \tilde{\mathbf{Y}}_{k}=\left[\begin{array}{llllll}
\tilde{\mathbb{F}}_{k} & \tilde{\Theta}_{k} & \tilde{\Phi}_{k} & \tilde{U}_{k} & \tilde{\mathbb{V}}_{k} & \tilde{\mathbb{W}}_{k}
\end{array}\right]^{T}, \\
& \tilde{\mathbf{Z}}_{k-1}=\left[\begin{array}{llllll}
\tilde{\mathbf{z}}_{1, k-1} & \tilde{\mathbf{z}}_{2, k-1} & \tilde{\mathbf{z}}_{3, k-1} & \tilde{\mathbf{z}}_{4, k-1} & \tilde{\mathbf{z}}_{5, k-1} & \tilde{\mathbf{z}}_{6, k-1}
\end{array}\right]^{T}
\end{aligned}
$$

\section{Results and Discussion}

\begin{tabular}{|c|c|c|c|c|}
\hline \multirow[b]{2}{*}{$G r^{\star}$} & \multicolumn{2}{|l|}{$-f^{\prime}(0,0)$} & \\
\hline & Singh\&Tewari [26] & Present & Singh\&Tewari [26] & Present \\
\hline 0.4 & 0.766 & 0.7656 & 0.400 & 0.4001 \\
\hline 1 & 0.618 & 0.6180 & 0.366 & 0.3656 \\
\hline 4 & 0.390 & 0.3904 & 0.298 & 0.2978 \\
\hline 6 & 0.333 & 0.3333 & 0.277 & 0.2767 \\
\hline 10 & 0.270 & 0.2701 & 0.251 & 0.2506 \\
\hline
\end{tabular}

In order to assess the validity and accuracy of the present numerical scheme, the results have been compared with that of previous work (Singh and Tiwari [26], Cheng [27]) in the absence of nonlinear convection parameters. It is found that they are in good agreement, as shown in Tables
Table 1: Comparison of $-f^{\prime}(0,0)$ and $-\theta^{\prime}(0,0)$ for various values of $G r^{\star}$ when $\mathcal{B}=0, \alpha_{1}=0, \alpha_{2}=0, n=1, \lambda=1, B i \rightarrow \infty$, and $\Omega=0$.

Table 2: Comparison of $-\theta^{\prime}(0,0)$ and $-\phi^{\prime}(0,0)$ for different values of $\mathcal{B}$ and Le when $G r^{\star}=0, \alpha_{1}=0, \alpha_{2}=0, n=1, \lambda=1, B i \rightarrow \infty$, and $\Omega=0$.

\begin{tabular}{c|c|cc|cc}
\hline & & $-\theta^{\prime}(0,0)$ & $-\phi^{\prime}(0,0)$ & \\
$\mathcal{B}$ & Le & Cheng [27] & Present & Cheng [27] & Present \\
\hline 4 & 1 & 0.9923 & 0.9923 & 0.9923 & 0.9923 \\
4 & 4 & 0.7976 & 0.7976 & 2.0550 & 2.0549 \\
4 & 10 & 0.6811 & 0.6810 & 3.2899 & 3.2898 \\
4 & 100 & 0.5209 & 0.5208 & 10.521 & 10.521 \\
1 & 4 & 0.5585 & 0.5585 & 1.3575 & 1.3575 \\
2 & 4 & 0.6494 & 0.6495 & 1.6243 & 1.6244 \\
3 & 4 & 0.7278 & 0.7278 & 1.8525 & 1.8524 \\
\hline
\end{tabular}

1 and 2. Therefore, the developed code can be used with great confidence to study the problem considered in this paper. The physical significance of the pertinent parameters such as $\xi, \alpha_{1}, \alpha_{2}, \Omega$ and $B i$ is determined through Figs. 2(a) to 6(c) for the boundary layer profiles. Also, the physical quantities of the present interest, skin friction, Nusselt and Sherwood numbers are projected in Figs.7(a) to 8 (c) for the same values. Here, the viscosity index $n$ ranged from 0.2 to 1.6 .

Variation of the non-dimensional velocity $f^{\prime}$, temperature $\theta$ and concentration $\phi$ across the boundary layer is presented in Figs. 2(a) to 2(c) for streamwise coordinate $\xi$. In a non-similar problem, the flow quantities change along the streamwise direction (in the present problem along the $x$-axis). Therefore, it is needed a non-dimensional parameter, $x$-dependent, which acts as a non-dimensional $x$-axis. This parameter determines the streamwise position where the flow quantities are calculated. When $\xi=0$, the flow governing equations are independent of streamwise location and hence, it shows that the existence of similarity solutions for the present problem. Increasing the value of streamwise coordinate $\xi$, the velocity component increases, whereas the thermal and solutal boundary layer thickness decreases. Further, the wall temperature always tends to 1 as $\xi \rightarrow \infty$. Hence, the changes in these profiles clearly proven that the present problem is non-similar. It 
means that the solutions are not unique for different values of $\xi$.

Figures 3(a) to 3(c) depicts the variations of momentum, thermal and solutal boundary layer profiles in the presence and absence of $\alpha_{1}$ for various values of $n$. As expected from Fig. 3(a), the increment in power-law index $n$ (from $n<1$ to $n>1$ ) leads to enhance the dimensionless velocity, due to the effect of shear-thinning in power-law fluids. In addition, the non-Newtonian fluid with a higher power-law index has a lesser thermal boundary layer thickness and a lesser concentration boundarylayer thickness, as shown in Figs. 3(b) and 3(c). So, increasing the power-law index tends to increase the flow and decrease the thermal boundary-layer and the concentration boundary layer thicknesses, thus increasing the heat and mass transfer rates from the plate. Increasing nonlinear temperature parameter $\alpha_{1}$ serves to enhance the velocity (Fig. 3(a)) on the surface of the inclined plate, whereas it takes reverse trend away from the plate. Physically, $\alpha_{1}>0$ implies that $T_{f}>T_{\infty}$; hence, there will be a supply of heat to the flow region from the wall and it accelerates the flow at the wall. An increase in $\alpha_{1}$ leads to reduce the temperature and concentration boundary layer profile in all three types of power-law indices and the same result projected in Figs. 3(b) and 3(c), respectively. Moreover the influence strength of $\alpha_{1}$ is gradually decreasing when power-law index is turned from $n<1$ to $n>1$.

The effect of the nonlinear concentration parameter on dimensionless velocity, temperature, and concentration profiles is shown in Figs. 4(a) to 4(c). The influence of nonlinear concentration parameter $\alpha_{2}$ ( $\alpha_{1}$ is fixed) on velocity reported similar behavior to that of $\alpha_{1}$. Thus, hydrodynamic boundary layer thickness is increased near to the surface for different values of $\alpha_{2}$. The effects of nonlinear concentration parameter on the thermal and solutal boundary layer flows are represented in Figs. 4(b) and 4(c). It is noticed from these figures that temperature and concentration profiles are decreased with increasing values of $\alpha_{2}$. However, the boundary layer thickness of the temperature and concentration are more in the absence of $\alpha_{1}$ and $\alpha_{2}$ in comparison with its presence. This is due to the enhancement of thermal and solutal density gradients by nonlinear terms in the momentum equation.

The significance of the Biot number $B i$ on the boundary layer profiles is illustrated by the Figs. 5(a) to 5(c) for different values of $n$. Figure 5(a) displays the variation of the velocity profile with or without Biot number. It is interesting to note that without Biot number (i.e. $B i=0$ ) the velocity is low. As the Biot number increases, the plate thermal resistance reduces. Consequently, the velocity in the neighborhood of the inclined plate increases signifi-

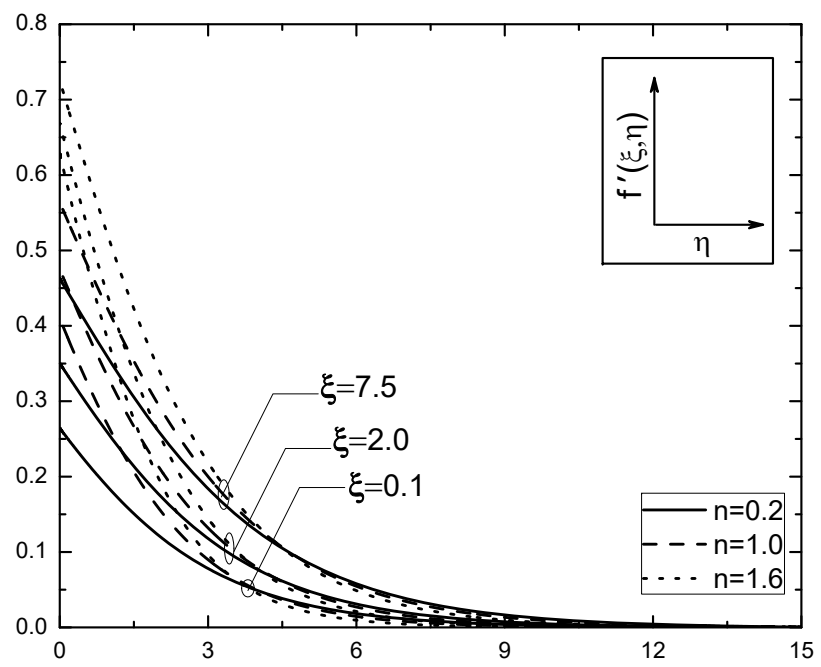

(a)

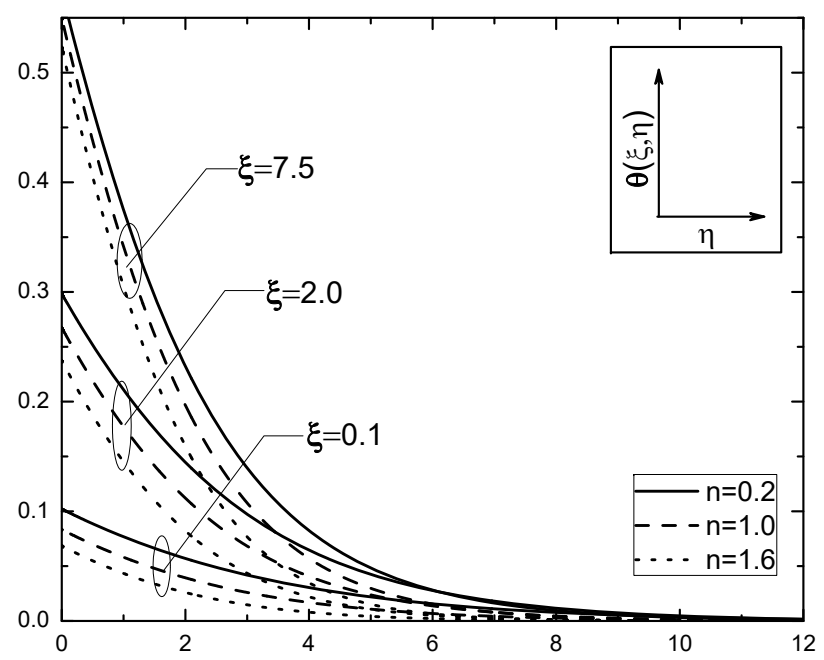

(b)

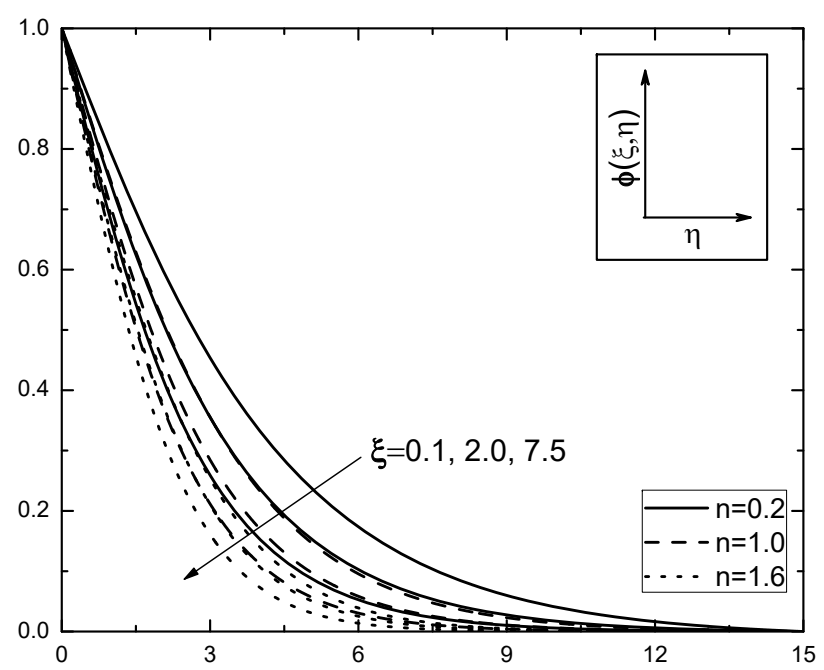

(c)

Fig. 2: Effect of $\xi$ for different values of $n$ on the $(a) f^{\prime}(\xi, \eta)$, (b) $\theta(\xi, \eta)$, and $(c) \phi(\xi, \eta)$ with the fixed values of $\alpha_{1}=1, \alpha_{2}=1$, $B i=0.2, \Omega=30^{\circ}$. 


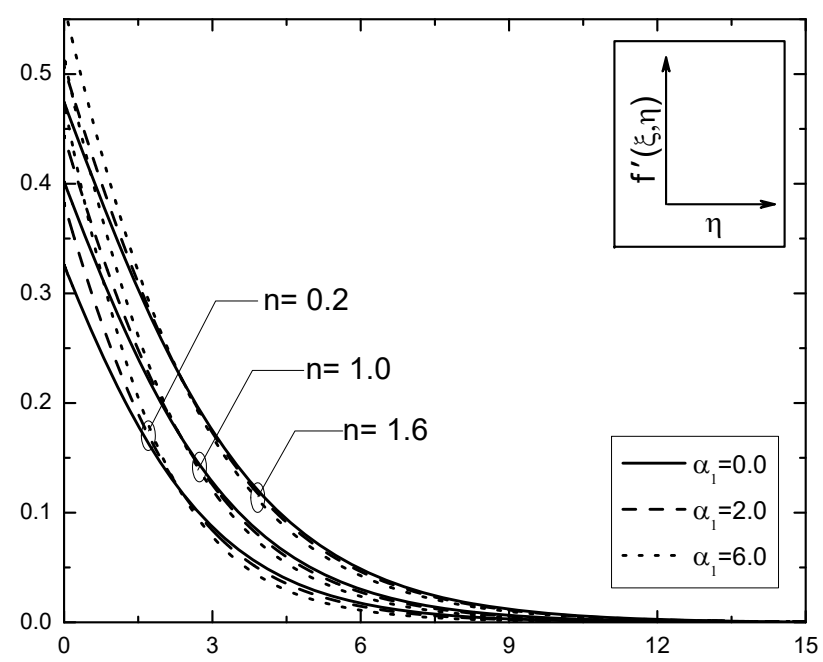

(a)

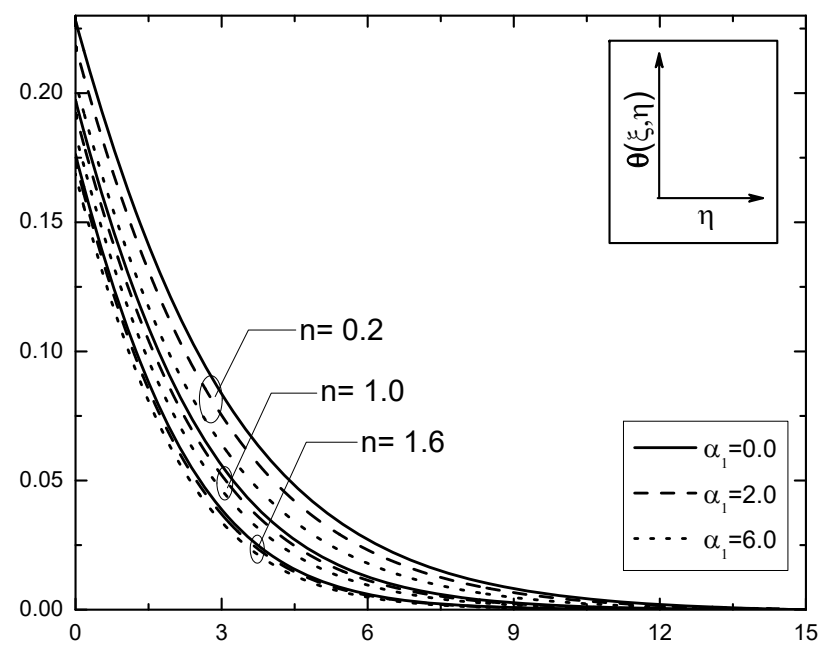

(b)

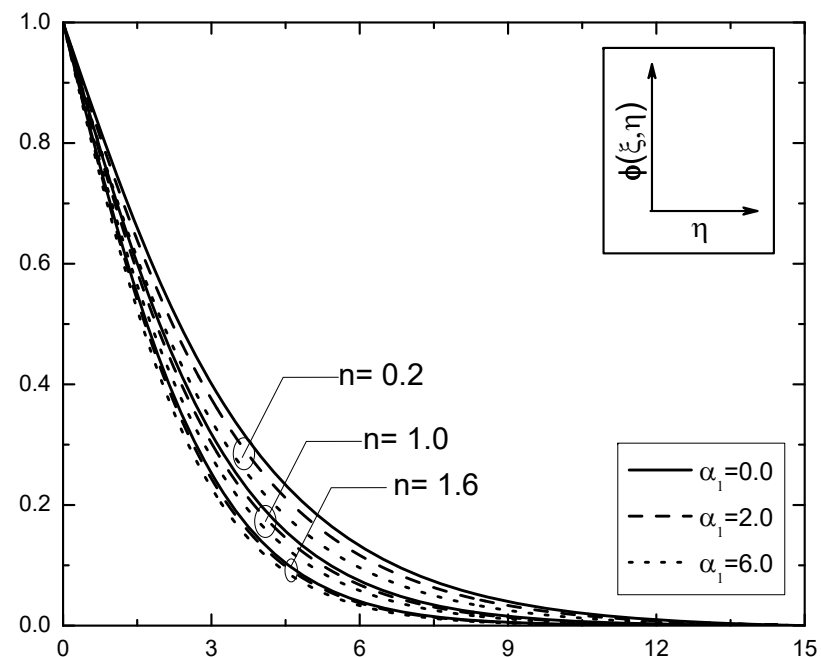

(c)

Fig. 3: Effect of $\alpha_{1}$ for different of values $n$ on the $(a) f^{\prime}(\xi, \eta)$, (b) $\theta(\xi, \eta)$, and $(c) \phi(\xi, \eta)$ with the fixed values of $B i=0.5, \alpha_{2}=1$, $\Omega=30^{\circ}, \xi=0.5$.

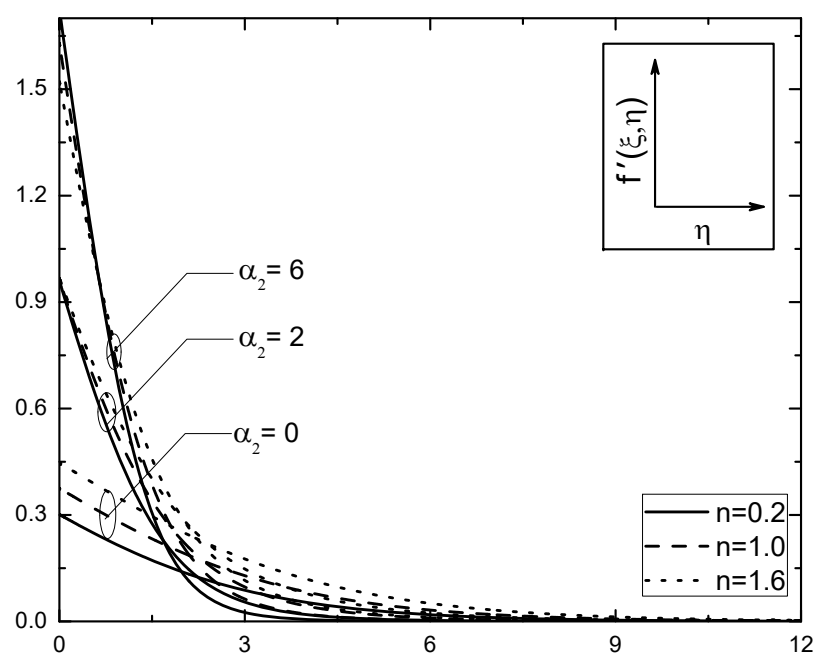

(a)

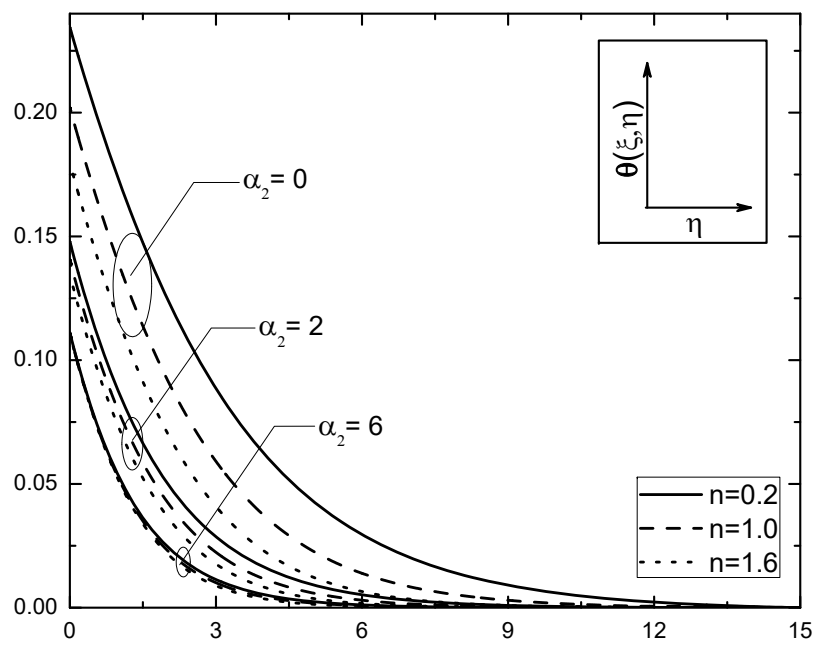

(b)

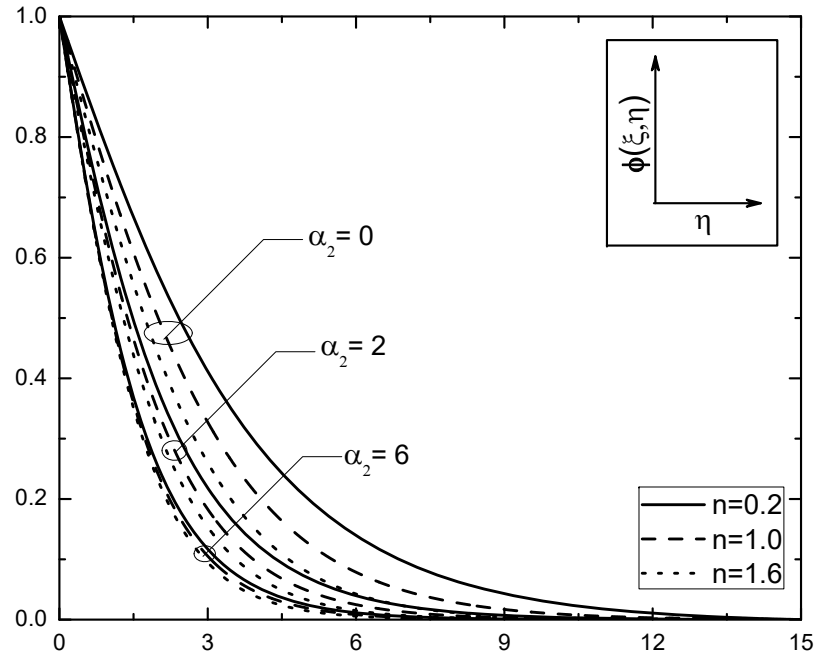

(c)

Fig. 4: Effect of $\alpha_{2}$ for different values of $n$ on the $(a) f^{\prime}(\xi, \eta)$, (b) $\theta(\xi, \eta)$, and $(c) \phi(\xi, \eta)$ with the fixed values of $B i=0.5, \alpha_{1}=1$, $\Omega=30^{\circ}, \xi=0.5$. 
cantly. Figs. 5(b) and 5(c) represent the effect of Biot number on the temperature and concentration profiles of the flow. It is evident that, as convective coefficient enhances from $B i<1$ (thermally thin case) to $B i>1$ (thermally thick case), the temperature of the flow increases whereas the concentration decreases. The convective boundary condition is the generalization of isothermal boundary condition and it effectively furnishes a mechanism for comparing the conduction resistance within a solid body to the convection resistance external to that body (offered by the surrounding fluid) for heat transfer. Since the isothermal boundary condition, $\theta(0)=1$ is a limiting result of the Biot number when $h_{f}$ tends to infinity (stated by Aziz [28]) and it is proven by Fig. 5(b). Usually, for high Biot number, the internal thermal resistance of the plate is high and the boundary layer thermal resistance is low. Further, when $B i=0$ (i.e. without Biot number) the left side of the plate with hot fluid is totally insulated, the internal thermal resistance of the plate is extremely high and no convective heat transfer to the cold fluid on the right side of the plate takes place. In this, the fluid temperature is maximum at the plate surface and decreases exponentially to zero value far out from the plate satisfying the boundary conditions, which is clearly observed from the Fig. 5(b). However, the temperature distribution is less in dilatants fluid and Newtonian fluid as compared to a pseudoplastic fluid. A similar observation was made by Khan and Gorla [29]. Further, the present results for temperature and concentration profiles are subjectively equal with the work of Makinde and Aziz [30].

The influence of the angle of inclination from the vertical, $\Omega$, ranging from $0^{0}$ to $90^{\circ}$, on the boundary layer profiles are displayed in Figs. 6(a) to 6(c). The physical reason for the depletion in velocity profile with respect angle of inclination is that the thermal and concentration buoyancy $\rho g^{\star} \cos \Omega$ (as considered in Eq. (9)) falls down when the angle $\Omega$ changed from $\Omega=0^{0}$ to $90^{\circ}$. The same illustration is noticed from Fig. 6(a). It is observed from Figs. 6(b) and 6(c) that the dimensionless temperature and concentration gradients $\theta$ and $\phi$ are enhances with increasing angle of inclination. Moreover, one can observe that the maximum buoyancy force for the same temperature difference and concentration difference occurs for $\Omega=0^{0}$ (vertical plate) and that there is no buoyancy force for the case $\Omega=90^{\circ}$ (horizontal plate), as the above term vanishes. In this case, the results of thermal and concentration distributions are identically equal to the work of Chamkha et al. [14], Chen [31].

In Fig. 7(a), the effects of the nonlinear convection parameters $\left(\alpha_{1}\right.$ and $\left.\alpha_{2}\right)$ are considered for different values of $n$ on skin friction (drag coefficient) against the streamwise

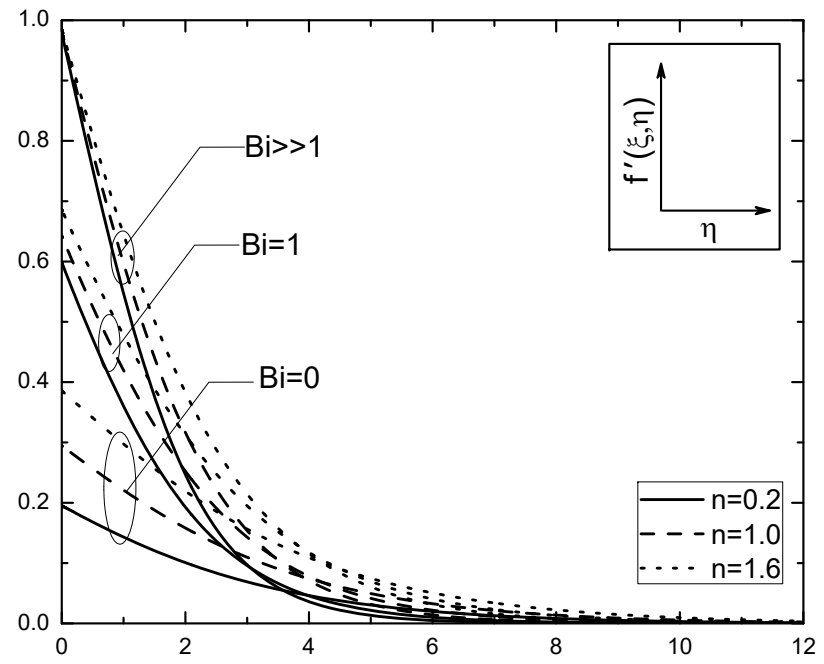

(a)

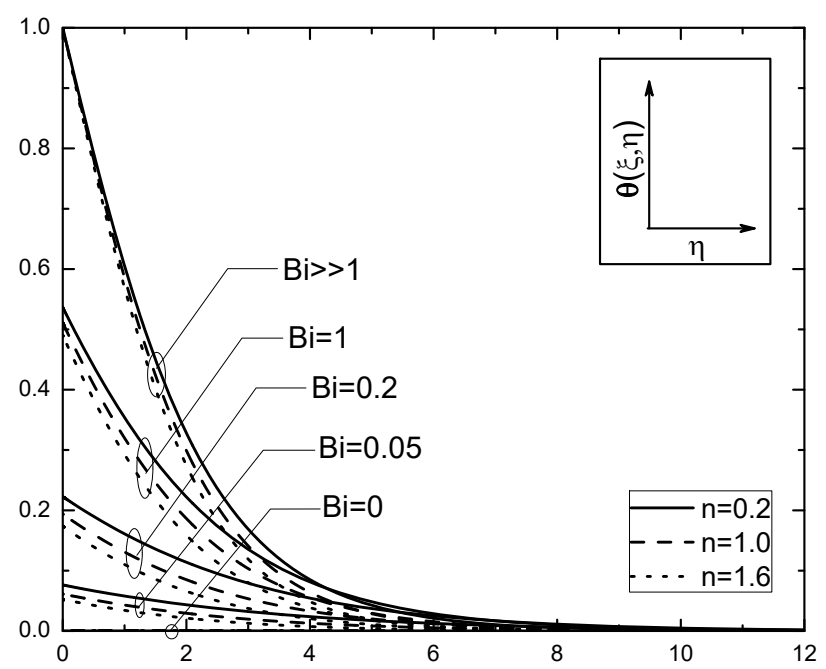

(b)

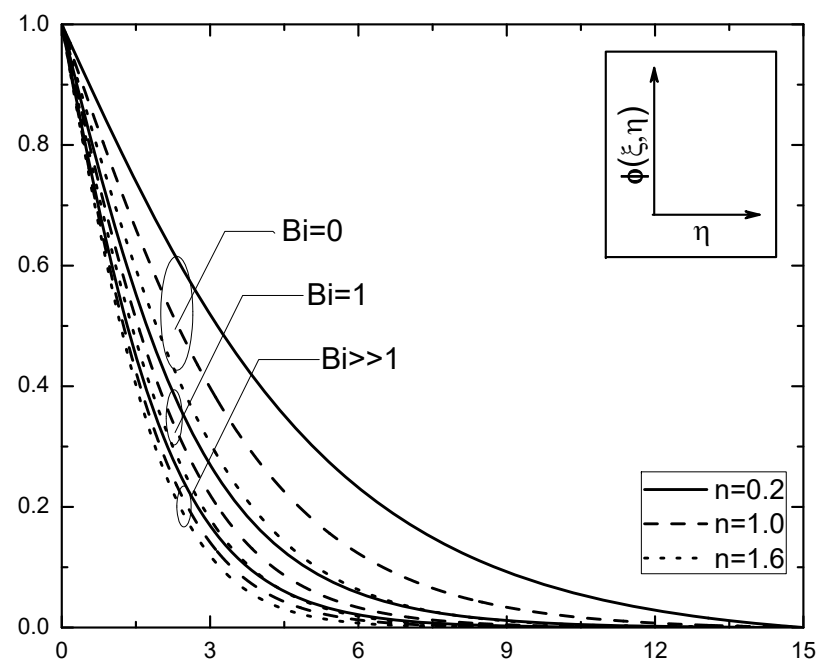

(c)

Fig. 5: Effect of Bi for different values of $n$ on the $(a) f^{\prime}(\xi, \eta)$, (b) $\theta(\xi, \eta)$, and $(c) \phi(\xi, \eta)$ with the fixed values of $\alpha_{1}=1, \alpha_{2}=1$, $\Omega=30^{\circ}, \xi=0.5$. 


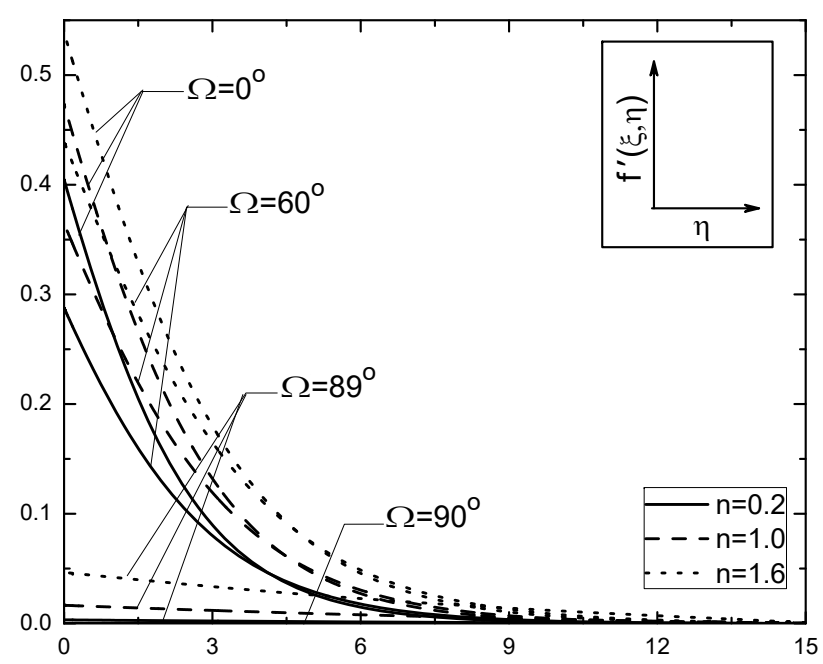

(a)

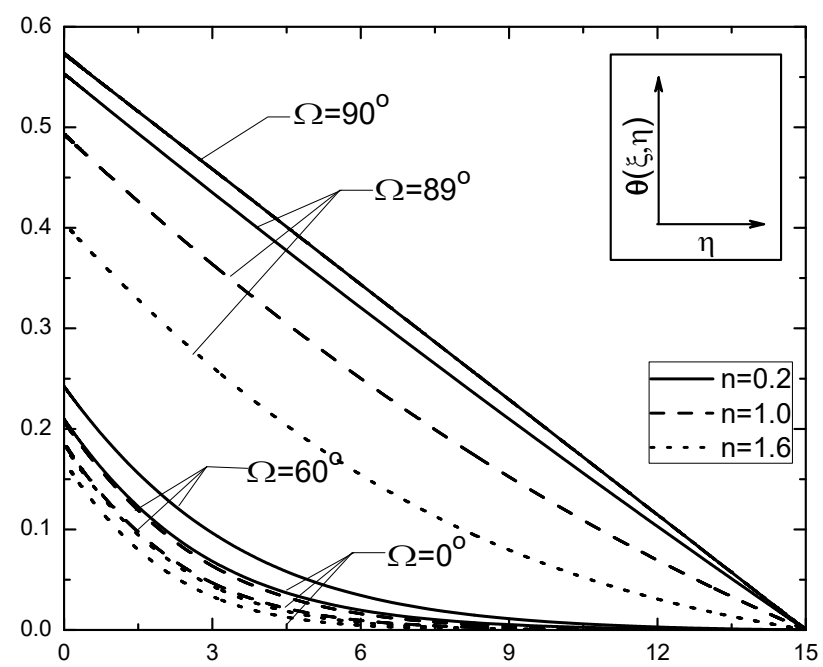

(b)

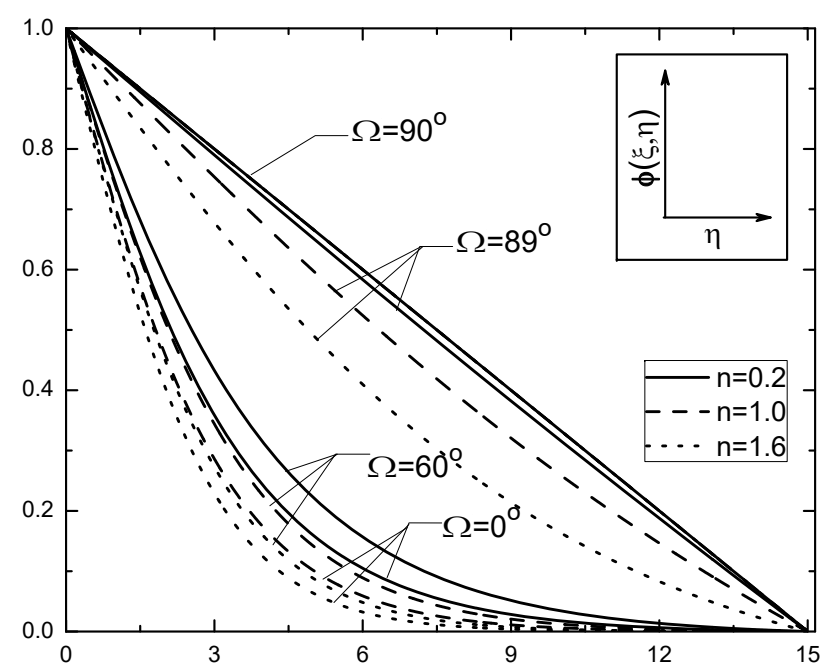

(c)

Fig. 6: Effect of $\Omega$ for different values of $n$ on the (a) velocity, (b) temperature, and (c) concentration with the fixed values of $\alpha_{1}=1$, $\alpha_{2}=1, B i=0.1, \xi=0.5$. coordinate $\xi$. It reveals that as power-law index increases, the skin friction increases. But with the enhancement of both nonlinear convection parameters the drag coefficient goes up. The power-law variation is more on the drag coefficient for the presence of both $\alpha_{1}$ and $\alpha_{2}$. The heat and mass transfer rates are evaluated against the streamwise coordinate for $\alpha_{1}$ and $\alpha_{2}$ in Figs. 7(b) and 7(c) together with power-law index $n$. Both the Nusselt and Sherwood numbers are increased with respect to power-law index in the presence or absence of nonlinear convection parameters. Along streamwise coordinate, both are increased and the influence of $\alpha_{2}$ is prominent as compared with $\alpha_{1}$. It is also observed that an increase in the value of the power-law index increases the value of the skin-friction coefficient, the Nusselt number, and the Sherwood number.

Effect of the Biot number and angle of inclination on the physical properties of the fluid flow are illustrated in Figs. 8(a) to 8(c) for different values of $n$. From Fig. 8(a), one can observe that an increase in the angle of inclination would produce a decrease in the buoyancy force and hence reduce the drag force for all values of $B i$, and which helps the flow past the plate and eases the mechanical processes of the flow through the medium. Also, the same kind of behavior is noticed for the Biot number changes, as shown in Fig. 8(a). Finally, Figs. 8(b) and 8(c) depict the effect of the dimensionless heat transfer rate and mass transfer rate, with an inclination angle $(\Omega)$ and Biot number $(B i)$. It is worth to mention that, as the Biot number increases from $B i<1$ (thermally thin case) to $B i>1$ (thermally thick case), evidently both the heat and mass rates are increases, as clearly seen in Figs. 8(b) and 8(c). Further, the dimensionless heat transfer rate and mass transfer rate both decrease with increasing inclination angle $\Omega$. Therefore, increasing the buoyancy enhances the heat and mass transfer rate to the plate (wall) which will lead to a decrease in temperature and concentration in the boundary layer, respectively. Thus, the heat and mass transfer rate are more for the case of vertical surfaces $\left(\Omega=0^{0}\right)$ as compared to horizontal surfaces $\left(\Omega=90^{\circ}\right)$.

\section{Conclusions}

In the present study, the nonlinear Boussinesq approximation is considered in the analysis of heat and mass transfer phenomena of an Ostwald-de Waele model powerlaw fluid flow over a convectively heated inclined plate in a non-Darcy porous medium. The influence of pertinent parameters on the velocity, temperature, concentration, skin friction, heat and mass transfer rates have been an- 


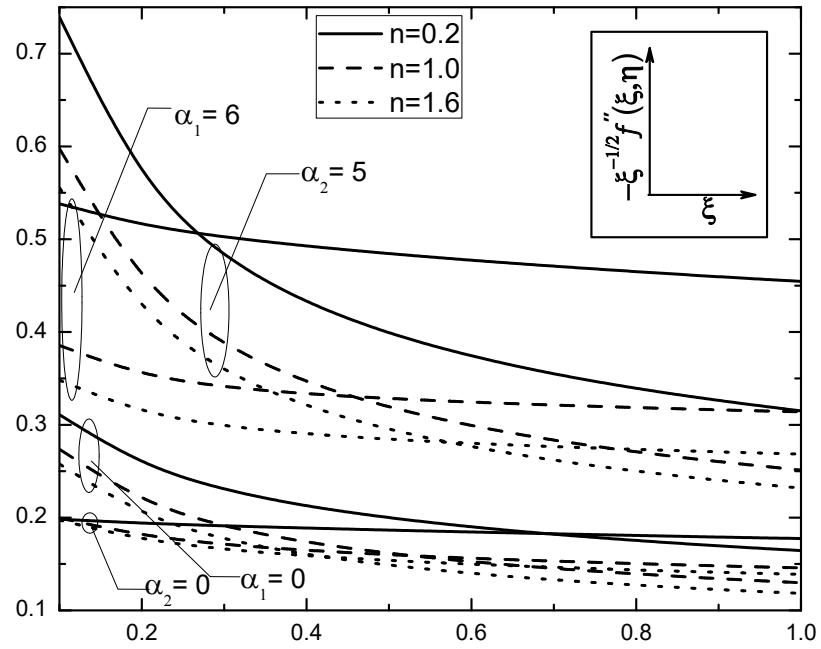

(a)

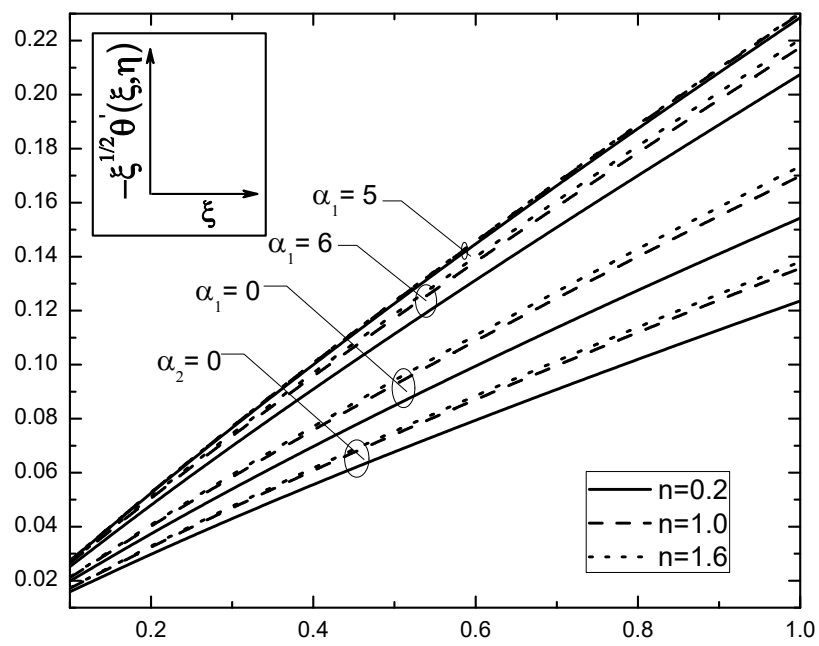

(b)

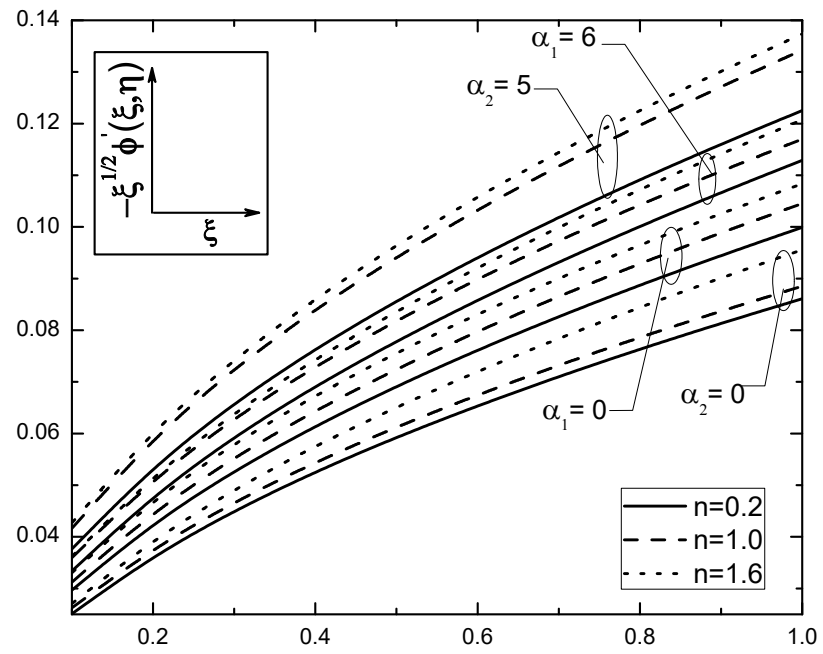

(c)

Fig. 7: Effect of $\alpha_{1}$ and $\alpha_{2}$ for different values of $n$ on the (a) skinfric tion, (b) Nusselt number, and (c) Sherwood number, against $\xi$ with the fixed values of $B i=0.5$ and $\Omega=30^{\circ}$.

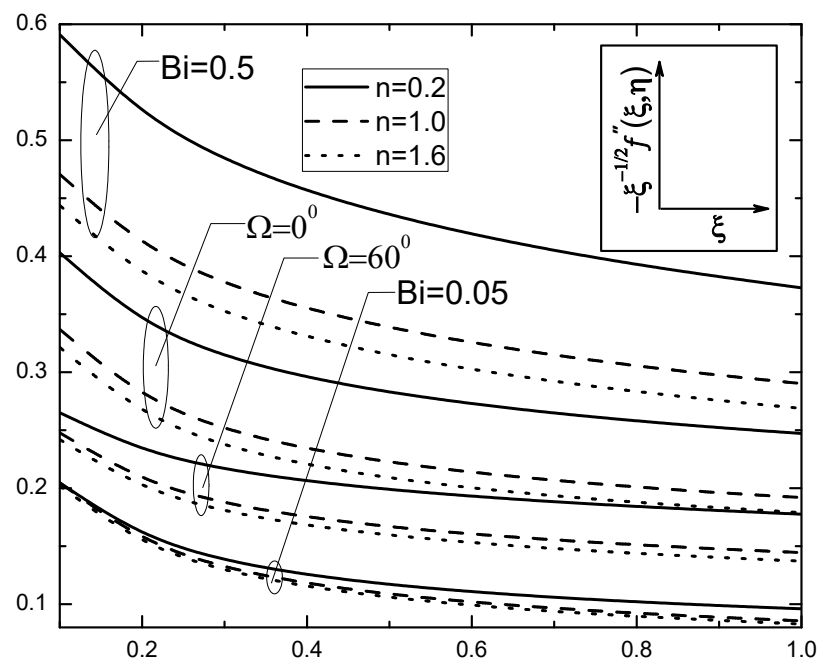

(a)

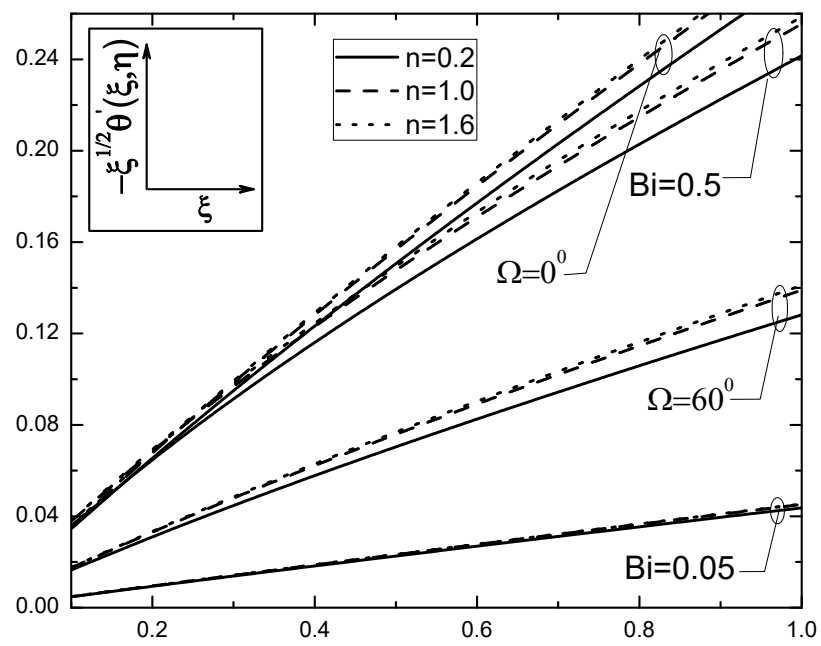

(b)

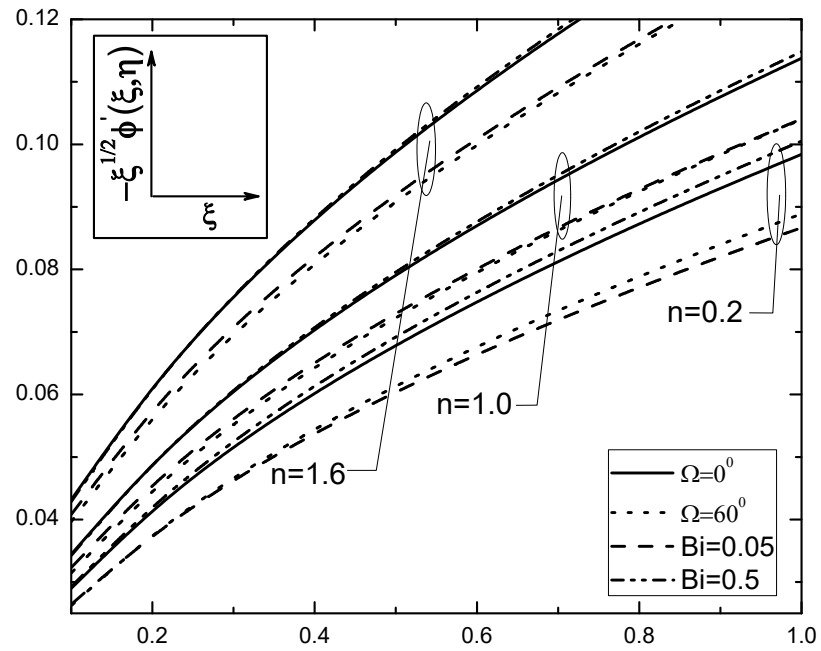

(c)

Fig. 8: Effect of Bi and $\Omega$ for different values of $n$ on the (a) skin friction, (b) Nusselt number, and (c) Sherwood number, against $\xi$ with the fixed values of $\alpha_{1}=1$ and $\alpha_{2}=1$. 
alyzed. The major notice is that the influence of $\alpha_{1}$ and $\alpha_{2}$ is prominent on all the physical characteristics of the present model, compared therewith its absence and these two effects are more influenced by pseudoplastic fluids. The variation of the Biot number leads to enhance all pertinent characteristics except the concentration profile. It effectively furnishes a mechanism for comparing the conduction resistance within a solid body to the convection resistance external to that body (offered by the surrounding fluid) for heat transfer. However, the velocity, heat, and mass transfer rates diminish, whereas the skin friction, thermal and solutal boundary layer thicknesses enhance with the increase of inclination of angle. This helps the flow past the plate and eases the mechanical processes of the flow through the medium. Also, this kind of investigation is useful in the mechanism of combustion, aerosol technology, high-temperature polymeric mixtures, solar collectors which are operated at moderate to very high temperatures.

Acknowledgement: This work was supported by of Council of Scientific and Industrial Research (CSIR), New Delhi, India (Project No 25 (0246)/15 /EMR-II).

\section{References}

[1] Shenoy AV. Darcy-Forchheimer natural, forced and mixed convection heat transfer in non-Newtonian power-law fluidsaturated porous media. Transport in Porous Media, 11(3), 219-241.

[2] Shenoy AV. Non-Newtonian fluid heat transfer in porous media. Advances in Heat transfer 1994, 24, 101-190.

[3] Subba Reddy Gorla R, Kumari M. Nonsimilar solutions for mixed convection in non-Newtonian fluids along a wedge with variable surface temperature in a porous medium. International Journal of Numerical Methods for Heat and Fluid Flow 1999, 9, 601-611.

[4] Kumari M, Nath G. Non-Darcy mixed convection in power-law fluids along a non-isothermal horizontal surface in a porous medium. International Journal of Engineering Science 2004, 42, 353-369.

[5] Cheng CY. Double-diffusive natural convection along a vertical wavy truncated cone in non-Newtonian fluid-saturated porous media with thermal and mass stratification. International Communications in Heat and Mass Transfer 2008, 35, $985-$ 990.

[6] Kairi RR, RamReddy C. Solutal dispersion and viscous dissipation effects on non-Darcy free convection over a cone in power-law fluids. Heat Transfer-Asian Research 2014, 43, 476-488.

[7] Makinde OD. On MHD heat and mass transfer over a moving vertical plate with a convective surface boundary condition. The Canadian Journal of Chemical Engineering 2010, 88, 983
990.

[8] Khan WA, Gorla RS. Nonsimilar solutions for mixed convection of water at $4^{\circ} \mathrm{C}$ over a vertical surface with a convection boundary condition in a porous medium. Heat Transfer-Asian Research. 2012, 41, 681-9.

[9] Ramzan M, Farooq M, Hayat T, Chung JD. Radiative and Joule heating effects in the MHD flow of a micropolar fluid with partial slip and convective boundary condition. Journal of Molecular Liquids 2016, 221, 394-400.

[10] Barrow H, Sitharamarao TL. Effect of variation in volumetric expansion coefficient on free convection heat transfer. British Chemical Engineering 1971, 16, 704-709.

[11] Vajravelu K, Sastri KS. Fully developed laminar free convection flow between two parallel vertical walls-I. International Journal of Heat and Mass Transfer 1977, 20, 655-660.

[12] Partha MK. Nonlinear convection in a non-Darcy porous medium. Applied Mathematics and Mechanics 2010, 31, 565574.

[13] Kameswaran PK, Sibanda P, Partha MK, Murthy PVSN. Thermophoretic and nonlinear convection in non-Darcy porous medium. Journal of Heat Transfer 2014, 136, 042601.

[14] Chamkha AJ, Issa C, Khanafer K. Natural convection from an inclined plate embedded in a variable porosity porous medium due to solar radiation. International Journal of Thermal Sciences 2002, 41, 73-81.

[15] Pal D, Chatterjee S. Soret and Dufour effects on MHD convective heat and mass transfer of a power-law fluid over an inclined plate with variable thermal conductivity in a porous medium. Applied Mathematics and Computation 2013, 219(14), 7556-7574.

[16] Murthy PVSN, Sutradhar A, RamReddy C. Double-diffusive free convection flow past an inclined plate embedded in a nonDarcy porous medium saturated with a nanofluid. Transport in porous media 2013, 98, 553-564.

[17] Murthy PVSN, Singh P. Heat and mass transfer by natural convection in a non-Darcy porous medium. Acta Mechanica 1999, 138(3-4), 243-254.

[18] Chen HT. Free convection flow of non-Newtonian fluids along a vertical plate embedded in a porous medium. Journal of Heat Transfer 1988, 110(1).

[19] Nield DA, Bejan A. Convection in porous media. SpringerVerlag, New York, 2013.

[20] Makukula ZG, Sibanda P, Motsa SS. A novel numerical technique for two-dimensional laminar flow between two moving porous walls. Mathematical Problems in Engineering 2010. http://dx.doi.org/10.1155/2010/528956

[21] Awad FG, Sibanda P, Motsa SS, Makinde OD. Convection from an inverted cone in a porous medium with cross-diffusion effects. Computers and Mathematics with Applications 2011, 61, 1431-1441.

[22] Khidir AA, Narayana M, Sibanda P, Murthy PVSN. Natural convection from a vertical plate immersed in a power-law fluid saturated non-Darcy porous medium with viscous dissipation and Soret effects. Afrika Matematika 2015, 26(7-8), 1495-1518.

[23] Yu HS, Sparrow EM. Local non-similarity thermal boundarylayer solutions. ASME Journal of Heat Transfer 1971, 93, 328334.

[24] Minkowycz W], Cheng P. Local non-similar solutions for free convective flow with uniform lateral mass flux in a porous medium. Letters Heat Mass Transfer 1982, 9, 159-168. 
[25] Canuto C, Hussaini MY, Quarteroni A, Zang TA. Spectral methods. Springer-Verlag, Berlin, 2006.

[26] Singh P, Tewari K. Non-Darcy free convection from vertical surfaces in thermally stratified porous media. International Journal of Engineering Science 1993, 31, 1233-1242.

[27] Cheng CY. Natural convection heat and mass transfer near a vertical wavy surface with constant wall temperature and concentration in a porous medium. International Communications in Heat and Mass Transfer 2000, 27, 1143-1154.

[28] Aziz A. A similarity solution for laminar thermal boundary layer over a flat plate with a convective surface boundary condition. Communications in Nonlinear Science and Numerical Simulation 2009, 14, 1064-1068.
[29] Khan WA, Gorla RSR. Heat and mass transfer in power-law nanofluids over a nonisothermal stretching wall with convective boundary condition. Journal of Heat Transfer 2012, 134, 112001.

[30] Makinde OD, Aziz A. Boundary layer flow of a nanofluid past a stretching sheet with a convective boundary condition. International Journal of Thermal Sciences 2011, 50, 1326-1332.

[31] Chen $\mathrm{CH}$. Heat and mass transfer in MHD flow by natural convection from a permeable, inclined surface with variable wall temperature and concentration. Acta Mechanica 2004, 172, 219-235. 